\title{
Feasible estimation in generalized structured models
}

\author{
Javier Roca-Pardiñas $\cdot$ Stefan Sperlich
}

Received: 25 February 2008 / Accepted: 5 May 2009 / Published online: 16 May 2009

(C) The Author(s) 2009. This article is published with open access at Springerlink.com

\begin{abstract}
This article introduces a feasible estimation method for a large class of semi and nonparametric models. We present the family of generalized structured models which we wish to estimate. After highlighting the main idea of the theoretical smooth backfitting estimators, we introduce a general estimation procedure. We consider modifications and practical issues, and discuss inference, cross validation, and asymptotic theory applying the theoretical framework of Mammen and Nielsen (Biometrika 90: 551-566, 2003). An extensive simulation study shows excellent performance of our method. Furthermore, real data applications from environmetrics and biometrics demonstrate its usefulness.
\end{abstract}

Keywords Generalized structured models $\cdot$ Smooth backfitting · Generalized varying coefficients · Generalized additive models $\cdot$ Computational statistics

\section{Introduction}

The curse of dimensionality is one of the major problems that arises when using nonparametric multivariate regres-

The authors thank Walter Zucchini and three anonymous referees for helpful comments and discussion. We acknowledge financial support from the Spanish Ministry of Education \& Science, MTM2008-03010, and Xunta de Galicia, PGIDIT07PXIB300191PR, and the Deutsche Forschungsgemeinschaft FOR916.

J. Roca-Pardiñas

Departamento de Estadística e I.O., Universidad de Vigo, c/Torrecedeira 86, 36208 Vigo, Spain

S. Sperlich ( $\varangle)$

Institut für Statistik und Ökonometrie, Georg-August Universität

Göttingen, Platz der Göttinger Sieben 5, 37073 Göttingen,

Germany

e-mail: stefan.sperlich@wiwi.uni-goettingen.de sion techniques. Therefore, much effort has been spent to reduce the dimensionality by imposing a certain structure, often yielding a semiparametric model. The resulting models can be divided in mainly two groups: one having a known structure that combines low or even one- dimensional smooth functions; the other possessing (semi)parametric indices combined by an unknown structure, e.g. having a nonparametric link function. They all compete for meeting the fundamental aspects, flexibility, dimensionality and interpretability, see Stone $(1985,1986)$. We concentrate here on the first group which has been called (inter alia) "generalized structured models", see Mammen and Nielsen (2003) or "semiparametric separable models", see Rodríguez-Poó et al. (2003).

While the latter gave a feasible estimation procedure and asymptotic theory it led to identification problems in several popular models. Mammen and Nielsen (2003) discussed the asymptotic theory of an abstract estimator but its implementation has been an unsolved problem so far. Hastie and Tibshirani (1990) introduced estimation procedures based on the backfitting algorithm for additive models, a particular member of our model class. Later, Opsomer and Ruppert (1997) and Opsomer (2000) developed asymptotic theory for this backfitting estimator. Several alternatives have been proposed to estimate (generalized) additive models, marginal impacts and interaction terms, see Binder and Tutz (2008) for various popular spline based methods, and Sperlich et al. (2002) for marginal integration.

Although many backfitting algorithms have had been developed since Friedman and Stuetzle (1981), the projection based smooth backfitting of Mammen et al. (1999) was probably the first that was accompanied by a complete asymptotic theory. Furthermore, despite its nontrivial derivation and implementation, it outperforms the existing procedures, see Nielsen and Sperlich (2005). Finally, in Mammen 
and Nielsen (2003) (see also Nielsen and Linton 1998) this projection idea has been applied to a general class of non and semiparametric regression, including time series models, with the common feature consisting of separable onedimensional smooth functions. However, while they have been able to derive some asymptotic theory, the calculation of those estimators has remained an open problem until now for most of the models discussed in that paper. Quite recently, Yu et al. (2008) proposed a smooth backfitting estimator for a generalized additive model and also provided a rigorous proof and a detailed discussion of a possible algorithm. It requires $d$-dimensional integration of a complex expression in each iteration.

In view of, first, the excellent performance shown in Nielsen and Sperlich (2005), second, its seemingly universal applicability discussed in Mammen and Nielsen (2003), and third, the need and benefit of feasible estimators for generalized structured models, we have developed a procedure for projection based estimators for this large class of models. In other words, we do not provide an alternative to the smooth backfitting but rather a practical omnibus procedure for an existing but theoretical estimator for a huge model class. We will see that the objective functions of all the different estimation problems - we speak of a huge class of rather different models-can be written in one compact formula. Moreover, other newly proposed smooth backfitting-estimators, e.g. for panel or non-stationary data, can be also written in our form. Finally, it can be easily applied to improve the estimation efficiency in models with nontrivial covariance structure, such as heteroscedasticity, panel data or time series. This latter aspect has not received much attention until now.

\subsection{The model class}

We consider a wide range of structured models. Using similar notation as Mammen and Nielsen (2003) we write the model class in its most general form as

$\Lambda(Y)=G\{Z, \beta, g(X)\}+S\{U, \gamma, s(T)\} \epsilon$

with $E[\epsilon \mid Z, X, U, T]=0$ for error $\epsilon$, i.e. $E[\Lambda(Y) \mid Z, X]=$ $G\{Z, \beta, g(X)\}$. Here, $\Lambda, G$ and $S$ are (up to a finite dimensional parameter) known functions, $\beta$ and $\gamma$ unknown finite dimensional parameter, and $g(\cdot), s(\cdot)$ unknown nonparametric functions; $Y$ is the dependent variable with observed covariates $(Z, X, U, T)$. In practice, often $(T, U) \subseteq(X, Z)$, but neither $Z \in \mathbb{R}^{q}$ has an overlap with $X \in \mathbb{R}^{d}$, nor $U \in \mathbb{R}^{p}$ with $T \in \mathbb{R}^{r}$. We impose further structure by setting

$$
\begin{aligned}
& g(X)=\left\{g_{0}, g_{1}\left(X_{1}\right), \ldots, g_{d}\left(X_{d}\right)\right\}, \\
& s(T)=\left\{s_{0}, s_{1}\left(T_{1}\right), \ldots, s_{r}\left(T_{r}\right)\right\} .
\end{aligned}
$$

This model class includes generalized partial linear additive and its sub-models,

$G\{Z, \beta, g(X)\}=\underline{G}\left\{Z^{t} \beta+g_{0}+\sum_{j=1}^{d} g_{j}\left(X_{j}\right)\right\}$

with $\underline{G}$ being the link,

partial linear varying coefficient models

$G\{Z, \beta, g(X)\}=\underline{G}\left\{g_{0}+\sum_{j=1}^{d} g_{j}\left(X_{j}\right) Z_{j}+Z_{\alpha}^{t} \beta\right\}$,

where $Z=\left(Z_{1}, \ldots, Z_{d}, Z_{\alpha}^{t}\right) \in \mathbb{R}^{d+q_{2}}, q=d+q_{2}$; but also models with multiplicative separability, or mixtures of both. In (4) often (but not necessarily) $X_{1}=\cdots=X_{d}$. While generalized additive and multiplicative models are quite popular in applications, for example in biostatistics, econometrics, environmetrics, technometrics, etc., varying coefficient models with nonparametric coefficients have been studied less. We will therefore give special attention to the latter, including a biometrical example. The estimation of the variance structure $S(\cdot)$, e.g. in visual GARCH and generalized CHARN models, is done in a second step working with residuals, after having predicted the mean $G(\cdot)$. These models are extremely popular in financial statistics but also in other econometric fields.

Apart from Yu et al. (2008), there exist other quite recent extensions: Mammen et al. (2006) have proposed a backfitting estimator for the panel model

$$
\begin{gathered}
Y_{i t}=\sum_{j=1}^{d} g_{j}\left(X_{j, i t}\right)+\eta_{t}+\lambda_{i}+\epsilon_{i t}, \\
i=1, \ldots, n, t=1, \ldots, T
\end{gathered}
$$

with $\eta_{t}, \lambda_{i}$ being fixed effects; Schienle (2007) introduced a consistent smooth backfitting procedure for additive models with non-stationary data; and Linton et al. (2008) proposed an estimator for the transformation model

$E[\Lambda(Y) \mid X]=g_{0}+\sum_{j=1}^{d} g_{j}\left(X_{j}\right)$,

where $\Lambda$ is known up to a finite dimensional parameter. Note that the models presented all fall into the class of (1), and therefore can be handled with our procedure.

\section{The general estimation procedure}

First we revise the main idea of smooth backfitting estimation concentrating on the local constant case for the sake of 
clearness; the local linear case is deferred to a computational note (Roca-Pardiñas and Sperlich 2008). Let us consider data with a single index $i$, i.e. given data $\left\{Y_{i}, Z_{i}, X_{i}\right\}_{i=1}^{n}$, and ignore the possibility of modelling the error variance. Then the smooth backfitting estimator is defined by

$$
\begin{aligned}
\hat{g}= & \underset{\bar{g}}{\arg \min } \int \sum_{i=1}^{n}\left[\Lambda\left(Y_{i}\right)-G\left\{Z_{i}, \beta, \bar{g}(x)\right\}\right]^{2} \\
& \times K_{h}\left(X_{i}-x\right) \pi d x
\end{aligned}
$$

with a product kernel $K_{h}(u)=\prod_{j=1}^{d} K_{h_{j}}\left(u_{j}\right), K_{h_{j}}\left(u_{j}\right)=$ $h_{j}^{-1} K\left(u_{j} / h_{j}\right)$ and bandwidth vector $h=\left(h_{1}, \ldots, h_{d}\right)$. Unless stated otherwise, we will denote by $h$ any bandwidth, no matter whether it is the $d$-dimensional vector or a subvector. The optional weight, denoted by $\pi$, typically depends on some of the covariates and will be discussed in detail below. The estimate of each $g_{j}$ is calculated given the other $g_{k}$ s, so we have to iterate over $j$.

As explained in detail in Nielsen and Sperlich (2005), for the additive model (3) one gets explicit expressions for each $\hat{g}_{j}$. They depend on one dimensional smoothers and on integrals containing two dimensional density estimates, i.e. for $\pi \equiv 1$

$\hat{g}_{j}\left(x_{1, j}\right)=\tilde{g}_{j}\left(x_{1, j}\right)-\sum_{k \neq j} \int \hat{g}_{k}\left(x_{k}\right) \frac{\widehat{p}_{j k}\left(x_{1, j}, x_{k}\right)}{\widehat{p}_{j}\left(x_{1, j}\right)} d x_{k}-\bar{Y}$,

where $\tilde{g}_{j}$ is the one dimensional Nadaraya-Watson smoother of $Y$ on $X_{j}$, and $\widehat{p}_{j k}\left(x_{1, j}, x_{k}\right), \widehat{p}_{j}\left(x_{1, j}\right)$ are the two-, respectively one-dimensional marginals of $\widehat{p}(x)$. However, switching from the additive to the varying coefficient model (4) the structure of the estimators no longer holds. The same arises to almost all other models.

More specific, allowing for a link function different from the identity, the minimization problem in (7) can no longer be solved directly. Instead, one has to use local scoring, which is analogous to iterative (re-)weighted least squares (McCullagh and Nelder 1989) for solving likelihood and nonlinear regression equations. At each iteration, an adjusted dependent variable is formed and a weighted local smoother is applied to fit the linearized transformed responses, see Roca-Pardiñas and Sperlich (2008) for details. The resulting effective objective function can be described as a weighted smooth backfitting algorithm.

When considering panel data, Mammen et al. (2006) introduce additional smoothing (denoted by $\pi$ ) along time. To get consistent backfitting estimates when the data are nonstationary, Schienle (2007) needs an additional trimming, which can be written in terms of a $0-1$ weighting function $\pi\left(X_{i}\right)$.

Xiao et al. (2003) considered efficient estimation in nonparametric regression with autocorrelated errors by a (prior) transformation of the response, and Vilar-Fernández and Francisco-Fernández (2002) introduced a weighted local linear estimator. We propose to combine these ideas with the smooth backfitting which then once again results in a weighted smooth backfitting with a transformed response. This is of particular interest for time series and panel data.

Note that the weights to which we are referring are either known up to a finite dimensional parameter (e.g. for parametric heteroscedasticity) or directly obtained from the procedure like in the local scoring algorithm. For mainly practical reasons we are not recommending a weighting done to reach asymptotic efficiency by nonparametrically estimated weights. (See also the next section.)

\subsection{A feasible estimator}

We state that the estimation procedure for all these models can be summarized in a common feasible minimization problem, namely

$$
\begin{aligned}
\underset{\bar{g}}{\operatorname{minimize}} \int & \sum_{i=1}^{n}\left[\tilde{Y}_{i}-m\left\{Z_{i}, \beta, \bar{g}(x)\right\}\right]^{2} W_{i} \\
& \times K_{h}\left(X_{i}-x\right) d x
\end{aligned}
$$

where $\tilde{Y}_{i}$ is the transformed or linearized (in local scoring) response and $m(\cdot)$ stands for a given pre-determined separable structure. For example, for the additive transformation model (6) we have $\tilde{Y}_{i}=\Lambda\left(Y_{i}\right)$ and $m\left\{Z_{i}, \beta, \bar{g}(x)\right\}=$ $g_{0}+\sum_{j=1}^{d} g_{j}\left(x_{j}\right)$. The weight $W_{i}$ can depend on $Z_{i}$, on $X_{i}$ or some other instruments like time, or even on $Y_{i}$. Clearly, $\pi$ from (7) is included in $W_{i}$ and will therefore no longer be mentioned separately. In all cases $W_{i}, \tilde{Y}_{i}$ should be easily available. As we will see below, in all cases considered, $W_{i}$ and $\tilde{Y}_{i}$ consist only of terms that are assumed to be known, easy to calculate, or are available from the estimation routine. Thus the proposed procedure is indeed feasible in practice.

Let us start with the example of estimating a varying coefficient model where $\beta=0$ and $\Lambda=G=$ identity. Then, the (weighted) smooth backfitting estimators $\left\{\hat{g}_{j}(\cdot), j=\right.$ $0, \ldots, d\}$ are the minimizers of

$$
\begin{aligned}
& \int \sum_{i=1}^{n}\left[\tilde{Y}_{i}-\bar{g}_{0}-\bar{g}_{1}\left(x_{1}\right) Z_{i 1}-\cdots-\bar{g}_{d}\left(x_{d}\right) Z_{i d}\right]^{2} \\
& \quad \times W_{i} \prod_{j=1}^{d} K_{h}\left(x_{j}-X_{i j}\right) d x
\end{aligned}
$$

with $\tilde{Y}_{i}=Y_{i}$. Under homoscedasticity one would set $W_{i} \equiv 1$. Some algebra gives that the solution is characterized by the 
following system of equations $(j=1, \ldots, d)$ :

$$
\begin{aligned}
\hat{g}_{j}\left(x_{j}\right)= & \frac{\sum_{i=1}^{n} \tilde{Y}_{i} Z_{i j} W_{i} K_{h}\left(x_{j}-X_{i j}\right)}{\widehat{p z}_{j}\left(x_{j}\right)} \\
& -\hat{g_{0}} \frac{\sum_{i=1}^{n} Z_{i j} W_{i} K_{h}\left(x_{j}-X_{i j}\right)}{\widehat{p z}_{j}\left(x_{j}\right)} \\
& -\sum_{k \neq j} \int \hat{g}_{k}\left(x_{k}\right) \frac{\widehat{p z}_{j k}\left(x_{j}, x_{k}\right)}{\widehat{p z}_{j}\left(x_{j}\right)} d x_{k}, \\
\hat{g_{0}}=\frac{1}{n} \sum_{i=1}^{n} \tilde{Y}_{i} & -\sum_{k=1}^{d} \int \hat{g}_{k}\left(x_{k}\right) \frac{1}{n} \sum_{i=1}^{n} Z_{i k} W_{i} K_{h}\left(x_{k}-X_{i k}\right) d x_{k},
\end{aligned}
$$

where $\widehat{p z}_{j}\left(x_{j}\right)=\sum_{i=1}^{n} Z_{i j}^{2} W_{i} K_{h}\left(x_{j}-X_{i j}\right)$

$$
\text { and } \begin{aligned}
\widehat{p z}_{j k}\left(x_{j}, x_{k}\right)= & \sum_{i=1}^{n} Z_{i k} Z_{i j} W_{i} K_{h}\left(x_{k}-X_{i k}\right) \\
& \times K_{h}\left(x_{j}-X_{i j}\right) .
\end{aligned}
$$

Note that for additive or multiplicative models we obtain estimators defined by similar expressions; for estimating (3) we only have to skip all $Z_{i j}, Z_{i k}$. The backfitting is necessary as the $\hat{g}_{k}, k \neq j$, on the left hand side are unknown. This leads to the iteration steps (with $Z_{i 0} \equiv 1$ )

$$
\begin{aligned}
\hat{g}_{j}\left(x_{j}\right)= & \underset{\bar{g}_{j}}{\arg \min } \int \sum_{i=1}^{n}\left[\tilde{Y}_{i}-\bar{g}_{j}\left(x_{j}\right) Z_{i j}\right. \\
& \left.-\sum_{k \neq j} \hat{g}_{k}^{\text {old }}\left(x_{k}\right) Z_{i k}\right]^{2} W_{i} K_{h}\left(x-X_{i}\right) d x
\end{aligned}
$$

yielding (11) to (14) with exclusively $\hat{g}_{k}^{\text {old }} \mathrm{s}$ on the left hand side.

Next, to account for a possible link $\underline{G} \neq$ identity, in generalized (partial linear) varying coefficients or additive models, the function $G(\cdot)$ and the variance $V(\cdot)$ are typically assumed to be known, so that the $W_{i}$ and $\tilde{Y}_{i}$ can be derived. For example, if the response $Y$ follows a distribution of the exponential family, the variance function $S^{2}(\cdot)=\varrho \cdot V(\cdot)$ is often assumed to depend on the covariates $X, Z$ through the same index $m_{i}:=m\left(Z_{i}, \beta, g\left(X_{i}\right)\right)$ as the mean function. When we minimize (7), here with $\Lambda=$ identity and given $\hat{\beta}$, local scoring leads to an estimation procedure where in each iteration for $\hat{g}_{j}$ we minimize

$$
\begin{aligned}
& \int \sum_{i=1}^{n}\left[\tilde{Y}_{i}-m\left(Z_{i}, \hat{\beta}, \hat{g}_{1}^{\text {old }}\left(x_{1}\right), \ldots, \bar{g}_{j}\left(x_{j}\right), \ldots, \hat{g}_{d}^{\text {old }}\left(x_{d}\right)\right)\right]^{2} \\
& \quad \times W_{i} \cdot K_{h}\left(X_{i}-x\right) d x
\end{aligned}
$$

with $\quad \tilde{Y}_{i}=\hat{m}_{i}^{\text {old }}+\left\{Y_{i}-\underline{G}\left(\hat{m}_{i}^{\text {old }}\right)\right\} / \underline{G^{\prime}}\left(\hat{m}_{i}^{\text {old }}\right), \quad W_{i}=$ ${\underline{G^{\prime}}}^{2}\left(\hat{m}_{i}^{\text {old }}\right) / V\left(\hat{m}_{i}^{\text {old }}\right)$, and $\hat{m}_{i}^{\text {old }}:=m\left(Z_{i}, \hat{\beta}, \hat{g}^{\text {old }}\left(X_{i}\right)\right)$. For example, for generalized varying coefficient models $m_{i}=$ $g_{0}+\sum_{j=1}^{d} g_{j}\left(X_{i j}\right) Z_{i j}$, and for generalized additive partial linear models, $m_{i}=g_{0}+\sum_{j=1}^{d} g_{j}\left(X_{i j}\right)+\beta^{T} Z_{i}$. The first order condition for $\hat{g}_{j}\left(x_{j}\right)$ again gives (11)-(14). Note that this necessitates an update of $W_{i}$ for nonlinear link functions.

By considering the other kinds of generalized structured model proposed by Mammen and Nielsen (2003), Rodríguez-Poó et al. (2003), Linton et al. (2008), Schienle (2007), Mammen et al. (2006), etc. one can check that we are indeed able to estimate all of these. While for models with nonlinear link the weight $W_{i}$ is a direct result of the local scoring algorithm, for non stationary models (Schienle 2007) it is a predetermined trimming. Mammen et al. (2006) propose a panel regression estimator with fixed time effects $\eta_{t}$, minimizing

$$
\begin{aligned}
& \sum_{i=1}^{n} \sum_{t=1}^{T} \int \sum_{s=1}^{T}\left\{Y_{i t}-\sum_{j=1}^{d} g_{j}\left(x_{j}\right)-\eta_{s}\right\}^{2} W_{s, t} \\
& \quad \times K_{h}\left(x-X_{i t}\right) d x,
\end{aligned}
$$

where the $W_{s, t}$ provide smoothing with respect to time and fulfilling $\sum_{s=1}^{T} W_{s, t}=1 \forall t$. Linton et al. (2008) propose an additive $\left(m_{i}=g_{0}+\sum_{j=1}^{d} g_{j}\left(X_{i j}\right)\right)$ transformation model using $W_{i}=1$ and $\tilde{Y}=\hat{\Lambda}(Y)$ with a pre-estimated parametric transformation $\Lambda$. Now, a straight-forward modification would be $m_{i}=g_{0}+\sum_{j=1}^{d} g_{j}\left(X_{i j}\right) Z_{i j}$. Also, (generalized) additive interaction models, as in Härdle et al. (2004) and in Rodríguez-Poó et al. (2003) (see their application example) are also become straight forward. Having obtained residuals from the fitted mean, $\varepsilon$, estimation of the variance function, $S(U, \gamma, s(T))$ (c.f. (1)), works in the same way, replacing $Y$ by $\varepsilon^{2}, G$ by $S$ etc. This is why we could claim in the introduction that our model class includes, for example, visual GARCH and generalized CHARN models.

Furthermore, with appropriate $W_{i}$ or $\tilde{Y}_{i}$, we may even improve the efficiency of the estimates, for example by taking the variance structure into account, see Sect. 2.2. For regression with autocorrelated errors, $\epsilon_{t}$, we propose to proceed as follows. Let $L$ be the lag operator and consider $\left(a_{0}-\sum_{s=1}^{\infty} a_{s} L^{s}\right) \epsilon_{t}=a(L) \epsilon_{t}=u_{s}$ with i.i.d. $u_{t}$ such that $a(L) Y_{t}=a(L) m\left\{Z_{t}, \beta, g\left(X_{t}\right)\right\}+u_{t}$. Clearly, the use of the infeasible $\tilde{Y}_{t}=Y_{t}-\sum_{s=1}^{\infty} a_{s}\left[Y_{t-s}-m\left\{Z_{t-s}, \beta, g\left(X_{t-s}\right)\right\}\right]$ would yield more efficient estimates. As can be derived from Xiao et al. (2003), we also have an efficiency gain when $m\left\{Z_{t-s}, \beta, g\left(X_{t-s}\right)\right\}$ is replaced by estimators obtained from a prior step using $Y_{t}$ instead of $\tilde{Y}_{t}$. The estimation of the $a_{s}$ is straight forward. An alternative treatment of AR structures (which, however, does not improve 
the first order properties of the final estimators) was proposed in Vilar-Fernández and Francisco-Fernández (2002).

We conclude by noting that, mathematically, (7) defines a theoretical estimator whereas the objective function (9) defines the computational procedure: Consider e.g. a nonlinear link function, then (7) defines the estimator, and (16) gives the algorithm.

\subsection{Remarks on modifications, statistical behavior, cross validation, and inference}

Recall that for models with nonlinear link $\underline{G}$ the weights have to be updated in each iteration. Therefore, it would be computationally attractive to get estimators of which the last term of (11), or $\widehat{p z}_{j}$ and $\widehat{p z}_{j k}$, do not depend on the weights $W$. Such alternatives are discussed in RocaPardiñas and Sperlich (2008). However, some of these were hard to justify from a theoretical point of view, and in some others require the computation of a $(d-1)$-dimensional integral of a nonparametric expression in each iteration step. In any case, some simulations gave clearly worse results than we obtained from the proposed procedure here, namely (9).

For the extension to weighted local linear smooth backfitting see Roca-Pardiñas and Sperlich (2008). Implementation and calculation is much more involved, but our simulations indicate a better performance.

Section 5 of Mammen and Nielsen (2003) provides the asymptotic framework, see also Mammen et al. (1999) for details in the additive model case. It is shown that, under the assumptions of twice differentiable components of $g(\cdot)$, partially differentiable $G$ (with partial derivatives denoted by $G_{j}$ ), covariates $X_{j}$ with densities $p_{j}$, errors $\epsilon$ with conditional mean zero and finite variance, and the typical regularity conditions on kernels and bandwidths $K_{h}$, the nonparametric components $\hat{g}_{j}$ converge with the one dimensional nonparametric rate to a normal distribution. The exact expressions of bias and variance depend on the model and the degree of the local polynomial. Therefore, rather general expressions for variance and bias are given but only for the case when $Z, \beta$ and variance function $S(\cdot)$ were dropped. The local linear version of our estimators are oracle efficient in the sense that neither bias nor variance of $\hat{g}_{j}, \hat{s}_{j}$ depend on the other components $\hat{g}_{k}, \hat{s}_{k}$, for $k \neq j$ if they exist. For the parametric part they show that, applying profiled projection techniques, $\beta$ can be estimated asymptotically as efficiently as if the nonparametric part $g(\cdot)$ were known, has the optimal parametric rate $\sqrt{n}$, and is asymptotically normal distributed.

It is interesting to note that competing classic backfitting estimators, if exist (we are only aware of algorithms and asymptotic theory for additive and generalized additive models), have larger bias. The marginal integration estimators have larger variance if the covariates are correlated.
If we make use of the possible transformation substituting $\tilde{Y}$ for $Y$ and/or including a weight function $W$ (respectively $\pi$ ), we can further increase the efficiency of smooth backfitting. Recall the case of autocorrelated errors or heteroscedasticity. For the first case we refer to Xiao et al. (2003), recall the end of Sect. 2.1; for the second we will briefly sketch the idea. Consider the case of $\Lambda, \underline{G}=$ identity and $\beta=0$. Then, the asymptotic variance of $\hat{g}_{j}\left(x_{j}\right)$, see Mammen and Nielsen (2003), is

$$
\frac{1}{n^{1 / 5} h_{j}} \frac{\int K^{2}(u) d u}{p_{j}\left(x_{j}\right)} E\left[(Y-m\{Z, g(X)\})^{2} \pi \mid X_{j}=x_{j}\right],(17)
$$

with $T=X, U=Z$. We see that choosing $\pi$, respectively $W$, inverse proportional to the error variance might improve the efficiency of $\hat{g}_{j}$. Certainly, the first order asymptotics can not improve for continuous $Z, X$ and $\sigma_{\epsilon}^{2}(Z, X)$ but (i) for discrete covariates (like gender) they can, and (ii) for finite samples the mean squared error can be reduced substantially even for continuous ones, compare Vilar-Fernández and Francisco-Fernández (2002). The conditions on the working weights depend on what one expects to achieve: while in theory one needs consistent estimators of the variance with a bias of rate $o\left(n^{-1 / 5} h_{j}^{-1}\right)$, in practice the proportions of the working weights should be similar to the optimal ones to avoid counter-efficient weighting. In other words, if the estimation of $W_{i}$ is as difficult as the main estimation problem, one should disregard asymptotic efficiency aspects, and use the simpler estimator.

Typical situations where such a weighting makes sense are parametric variance functions, in particular heteroscedasticity by factors allowing for clustering. For example, in econometrics we often observe different residual variances for males vs. females, see Sect. 3.1, where for $W^{-1}=\widehat{\sigma}$ (gender) we got rather encouraging results. The incorporation of nonparametric volatility functions in $W_{i}$ can also be useful if the structure is reasonably simple. For growth models (in macro-econometrics or biometrics) exponential specifications are quite sensible. With the assumption that the volatility is a function of the mean we get $\operatorname{Var}\left[Y_{i} \mid X_{i}, Z_{i}\right]=\sigma^{2} \exp \left\{\alpha m_{i}\right\}$ with unknown $\sigma^{2}, \alpha$. An alternative is the assumption of a constant coefficient of variation. Note that an inverse weighting with the latter specification can not improve the first order asymptotics and thus only makes sense for small and moderate sample sizes.

Sometimes, however, the inclusion of $W_{i}$ 's is due to computational needs but not to an additional weighting $\pi$ in (7); for example when we face nonlinear link functions $\underline{G}$, it results from local scoring. Then, it is not subject to additional assumptions.

Although we use asymptotic expressions to discuss efficiency aspects, they are not sufficiently exact in practice for doing reliable inference. We recommend resampling methods instead. Bootstrap inference in generalized 
additive partial linear models has been studied extensively in Härdle et al. (2004), in particular for the construction of confidence bands, but also for testing of parametric specifications and interactions. They introduced bootstrap procedures for different model specifications (see their Sect. 2.2). At that stage they do not make use of a possible additive structure of the nonparametric part so that it is straight forward to see how their methods apply for our general model class. Furthermore, Roca-Pardiñas and Sperlich (2007) introduced and compared eleven test statistics for generalized additive models, always using bootstrap to determine the critical values. Neither their bootstrap nor their test statistics made particular use of the separability structure so that, again, they can also be applied to our general model class. Summarizing, we propose to use bootstrap for estimating variances, testing, and to construct confidence intervals or bands, see Sect. 3.2.2.

Rodríguez-Poó et al. (2007) developed an adaptive omnibus test for the class of weakly separable models which is basically our model class. To check a possible misspecification they proposed to test $H_{0}: m(z, x)=m_{0}(z, x) \forall(z, x)$ from the support of $(Z, X)$, where $m$ is now a shortcut for the mean function. For the construction of the test statistic they defined first

$I_{h_{t}}=\int\left[\frac{1}{n h_{t}^{d}} \sum_{j=1}^{n} L\left(\frac{x-X_{j}}{h_{t}}\right)\left(Y_{j}-\widehat{m}\left(Z_{j}, X_{j}\right)\right)\right]^{2} d x$

with a $d$-dimensional kernel $L$ and a test bandwidth $h_{t}$. As this statistic will vary with $h$, they took the standardized version

$T_{h_{t}}=\frac{n h_{t}^{d / 2} I_{h_{t}}-h_{t}^{-d / 2} \hat{B}}{\sqrt{\hat{V}}}$

where $\hat{B}$ is an estimator of the expectation of $n h_{t}^{d / 2} I_{h_{t}}$ under $H_{0}$, and $\hat{V}$ an estimator of its variance. The final test statistic proposed was $T^{*}=\max _{h_{t} \in H_{n}} T_{h_{t}}$ with $H_{n}$ being a predetermined finite set of possible testing bandwidths. Evidently, if one is not interested in an adaptive test but rather focusing on a certain smoothness (given $h_{t}$ ), one can use $I_{h_{t}}$ directly for testing $H_{0}$. In any case the critical values were calculated via bootstrap or subsampling. Rodríguez-Poó et al. (2007) provide a complete asymptotic theory and also simulation studies, but based on estimators that are different to ours. The development of more specific tests on separability, specific interactions, parametric forms, etc. and its asymptotic theory is quite involved and deferred to future research.

Cross validation is useful for measuring prediction power, model or bandwidth selection. We consider the last mentioned problem, and for this purpose we use the notation $h=\left(h_{1}, \ldots, h_{d}\right)$, thus allowing for different bandwidths for each $g_{j}, j=1, \ldots, d$. In order to find the optimal $h$ we propose to minimize the cross validation criterion

$C V_{j}\left(h_{j}\right)=\sum_{i=1}^{n} W_{i}\left\{\tilde{Y}_{i}-\bar{m}_{i}\left(h_{j}\right)\right\}^{2}$,

for each direction $j$. Here, $\bar{m}_{i}\left(h_{j}\right)$ is an estimator of $m_{i}$ but where the first summand of $\hat{g}_{j}$, i.e. the weighted NadarayaWatson estimator in (11), does not use the $i$ th observation. The added $\left(h_{j}\right)$ highlights its dependency on bandwidth $h_{j}$.

\section{Finite sample performance}

We will concentrate on four different models: generalized (partial linear) additive model with binary response, a generalized varying coefficients model with binary response, and a partial linear additive model with continuous response under heteroscedasticity. Since, to the best of our knowledge, competing procedures exist only for the particular additive case we have included a simulation comparison with other estimators for generalized additive models. Afterwards, we apply our procedure to two different real data problems. In contrast to the rest of our model class, generalized additive models have been being studied intensively, though with other methods so far. Therefore we give a special emphasis on generalized varying coefficient models, including the construction of confidence bands (Sect. 3.2.2). All calculations have been done with the use of linear binning ${ }^{1}$ and, if not mentioned otherwise, with Cross Validation bandwidths. Mean squared errors are calculated from 1000 simulations.

\subsection{Simulation examples}

We start with a generalized additive model with binary response,

$P(Y=1 \mid X)=\frac{\exp \left\{\sum_{j=1}^{d} g_{j}\left(X_{j}\right)\right\}}{1+\exp \left\{\sum_{j=1}^{d} g_{j}\left(X_{j}\right)\right\}}$.

The covariate vector $X=\left(X_{1}, \ldots, X_{d}\right)$ was generated us$\operatorname{ing} X_{j}=3 \arctan \left(\tilde{X}_{j}\right) / \pi$ with $\tilde{X}_{j} \sim N(0,1)$ and $\rho=\rho_{j k}=$ $\operatorname{Corr}\left(\tilde{X}_{j}, \tilde{X}_{k}\right)$ for $j \neq k$. This link gives a logit model; the arctan transformation is to have the covariates mainly in $[-1,1]$; and the normality of $\tilde{X}$ is to control the dependence of the covariates $X_{j}$ via the correlation $\rho$. The dependence is a parameter to control the complexity of the estimation

\footnotetext{
${ }^{1}$ The simple binning assigns to each grid point a weight equal to the number of observation in its bin. In the so-called linear binning this weight is the sum of inverse relative distances between the observations and the two closest grid points, see Fan and Marron (1994) and Wand (1994).
} 
Fig. 1 The $\operatorname{MSE}\left(\hat{h}_{\text {opt }}\right)$ with 1 -sigma-bands, and $d \cdot \operatorname{MSE}_{1}\left(\hat{h}_{\text {opt }}\right)$ (straight line $)$ as functions of $\rho ; n=400$ and $d=4$ in model (20) with $g_{j}(x)=2 \sin (\pi x)$ for $j=1, \ldots, 4$

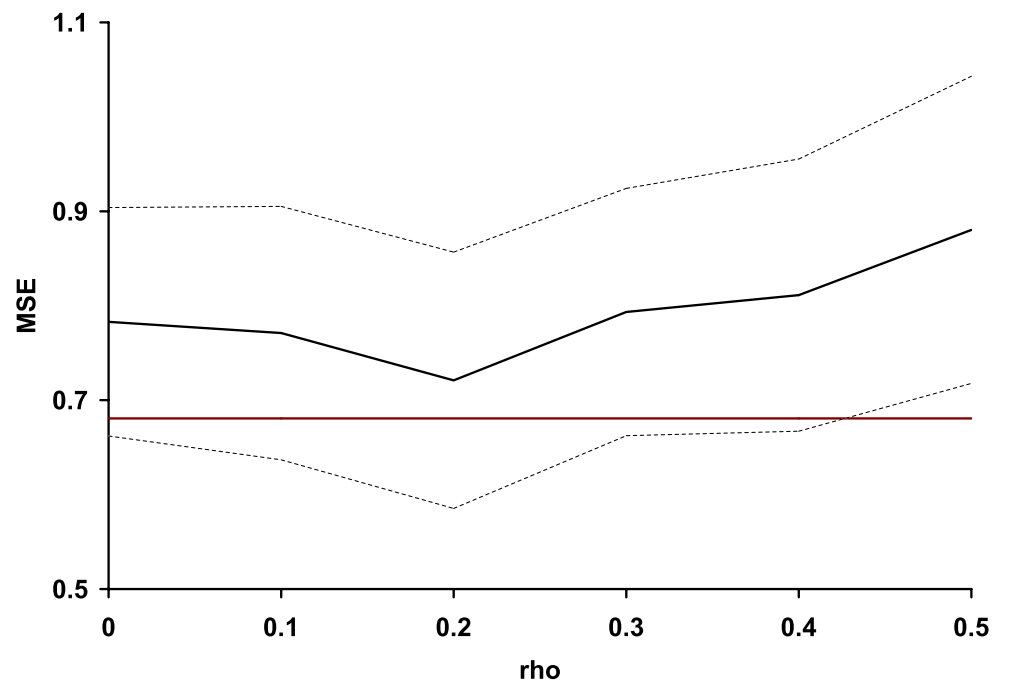

problem, see Sperlich et al. (1999) or Nielsen and Sperlich (2005).

The first exercise is to see how much the sample average mean squared error of the index function, ${ }^{2}$ defined by $\frac{1}{n} \sum_{i=1}^{n} E\left[m_{i}-\widehat{m}_{i}\right]^{2}$ and denoted by $\operatorname{MSE}\left(\hat{h}_{\text {opt }}\right)$, increases for increasing $\rho$. In order to check this we generated samples of size $n=400$ as described above with $d=4$ and $g_{j}\left(x_{j}\right)=2 \sin (\pi x)$ for all $j$. In Fig. 1 we see the $\operatorname{MSE}\left(\hat{h}_{\text {opt }}\right)$ as a function of $\rho$ together with simple $\sigma$-bands and $d$ times the MSE of the corresponding one-dimensional problem, i.e. $d \cdot \operatorname{MSE}_{1}\left(\hat{h}_{\text {opt }}\right)$ (straight line) for comparison. Even though the 4-dimensional problem has larger errors, it is surprising how well the estimator does compared to the onedimensional estimation problem even for strongly dependent covariates.

In order to increase the dimension, we added $g_{5}\left(x_{5}\right)=x_{5}$ and $g_{6}\left(x_{6}\right)=-x_{6}$, fixed $\rho=0.25$ and tried several sample sizes $n$. Note that this model can be estimated directly as a generalized partial linear additive model with $\left(Z_{1}, Z_{2}\right)=$ $\left(X_{5}, X_{6}\right)$ and $\left(\beta_{1}, \beta_{2}\right)=(1,-1)$. How the $\operatorname{MSE}\left(\hat{h}_{\text {opt }}\right)$ converges with $n$ can be seen in Fig. 2. There we see that the average mean squared error indeed converges at its theoretical rate $n^{-4 / 5}$, but that it does even better, at least in the distri-

${ }^{2}$ For the generalized additive model with $m(X)=\sum_{j=1}^{d} g_{j}\left(X_{j}\right)$, given a sample $\left\{\left(\mathbf{X}_{i}, Y_{i}\right)\right\}_{i=1}^{n}$ from (20), the $\operatorname{MSE}\left(\hat{h}_{\text {opt }}\right)$ has been defined by $\operatorname{MSE}=1 / n \sum_{i=1}^{n}\left\{\sum_{j=1}^{d} \hat{g}_{j}\left(X_{i j}\right)-\sum_{j=1}^{d} g_{j}\left(X_{i j}\right)\right\}^{2}$,

and for the generalized varying coefficients model with $m(X, Z)=$ $\sum_{j=1}^{d} \alpha_{j}\left(X_{j}\right) Z_{j}$ by

$\operatorname{MSE}=1 / n \sum_{i=1}^{n}\left\{\sum_{j=1}^{d} \hat{\alpha}_{j}\left(X_{i j}\right) Z_{i j}-\sum_{j=1}^{d} \alpha_{j}\left(X_{i j}\right) Z_{i j}\right\}^{2}$. bution sense of getting a much smaller interquartile range. These two first simulations demonstrate that the weighted smooth backfitting overcomes the curse of dimensionality completely, in theory and practice.

Next we come to a model that is less known, and we are not aware of any competing procedure: the generalized varying coefficients models with binary response,

$P(Y=1 \mid Z, X)=\frac{\exp \left\{\sum_{j=1}^{3} \alpha_{j}\left(X_{j}\right) Z_{j}\right\}}{1+\exp \left\{\sum_{j=1}^{3} \alpha_{j}\left(X_{j}\right) Z_{j}\right\}}$.

The covariates $X_{1}, X_{2}$ and $X_{3}$ were chosen as independent random variables, uniformly distributed at $[1,4]$. Covariates $Z_{1}$ and $Z_{2}$ were generated independently in accordance with $Z_{1} \sim \operatorname{Bernoulli}(0.4), Z_{2} \sim N(0,1)$, and $Z_{3}$ was set to $Z_{3}=Z_{2}^{2}$. For our simulation study we set $\alpha_{1}\left(x_{1}\right)=\sqrt{x}_{1}$, $\alpha_{2}\left(x_{2}\right)=\log \left(x_{2}\right)$, and $\alpha_{3}\left(x_{3}\right)=1$.

The performance was again measured by the sample average of mean squared errors of the index function. In Fig. 3 we multiplied them by the theoretical rate $n^{4 / 5}$. The figures confirm the nice performance of our estimation procedure.

A further exercise was to see how much better the weighted smooth backfitting performs compared to the classic smooth backfitting without weighting in the presence of unknown heteroscedasticity in an additive regression model

$Y=\sum_{j=1}^{4} g_{j}\left(X_{j}\right)+Z_{1}+Z_{2}+\epsilon\left(Z_{2}\right)$,

with $X_{1}$ to $X_{4}$ and $g_{1}$ to $g_{4}$ as in the first exercise (20), where $\rho=0.25, Z_{1} \sim \operatorname{Bernoulli}(0.4), Z_{2} \sim \operatorname{Bernoulli}(0.6)$, and $\epsilon\left(Z_{2}\right) \sim N\left(0, \sigma^{2}\left(Z_{2}\right)\right)$ with $\sigma(0)=4, \sigma(1)=2$, and set $n=500$. A typical example from practice would be $Z_{2}=$ gender. Again we are not aware of any alternative to our estimation procedure. 
Fig. 2 The $n^{4 / 5} \operatorname{MSE}\left(\hat{h}_{\text {opt }}\right)$ as functions of $n ; \rho=0.25$ and $d=6$ in model (20)
Fig. 3 The $n^{4 / 5} \operatorname{MSE}\left(\hat{h}_{o p t}\right)$ as functions of $n$ in model (21)
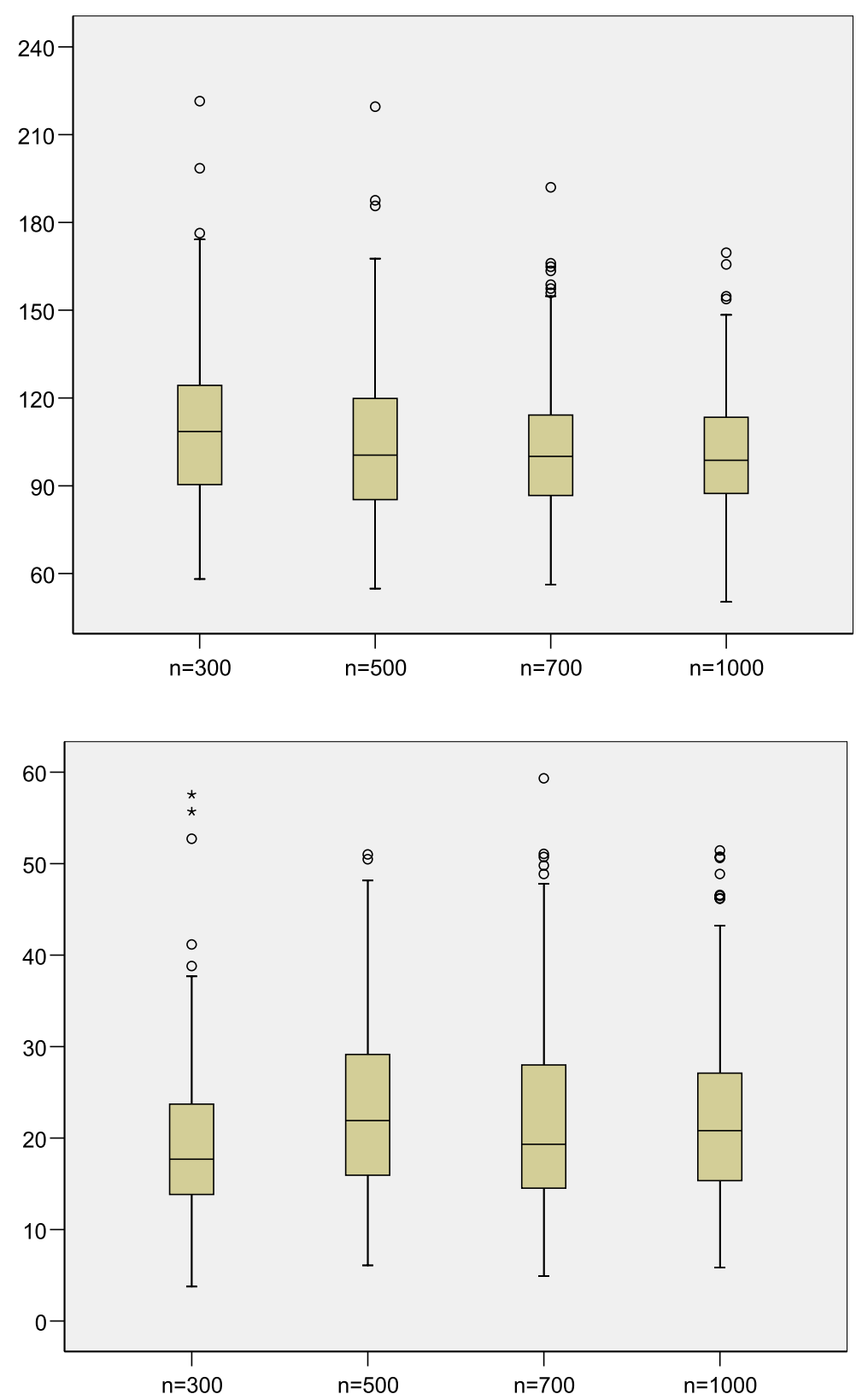

We first ran a simple smooth backfitting with cross validation bandwidths giving a MSEn $n^{4 / 5}$ of 117 with standard deviation 22. From the residuals we estimated $\sigma(0)$ and $\sigma(1)$, set $W_{i}=\widehat{\sigma}^{-2}\left(Z_{i, 2}\right)$ and did the weighted smooth backfitting. This gave a MSEn $n^{4 / 5}$ of 88 with standard deviation 17, see Fig. 4. We see a clear and substantial efficiency gain thanks to our weighting. Note that the results would favor our weighted version even much more if $Z_{2}$, the variable causing the heteroscedasticity, were correlated with the other covariates, something quite typical in practice.

The next exercise is to compare the weighted smooth backfitting with alternative estimators. However, while we are able to estimate with our procedure a large class of rather different models, see (1), we are aware of competing estimators only for the particular case of the well studied generalized additive models. Actually, to date, several contributions to GAMs can be found in the literature. Hastie and Tibshirani (1990) discussed various approaches using smoothing splines. Wahba et al. (1995) proposed the use of smoothing spline analysis-of-variance methods, and Wood (2003) introduced a numerical procedure based on regression splines. Nowadays, there exists standard software like the mgcv package in $\mathrm{R}$, to fit this model.

We compared the local constant smooth backfitting with the classical backfitting estimator of Hastie and Tibshirani (1990) with local constant kernel smoothers, its modified 
Fig. 4 The $n^{4 / 5} \operatorname{MSE}\left(\hat{h}_{\text {opt }}\right)$ of the non weighted (left) versus the weighted (right) smooth backfitting in a heteroscedastic additive partial linear regression model

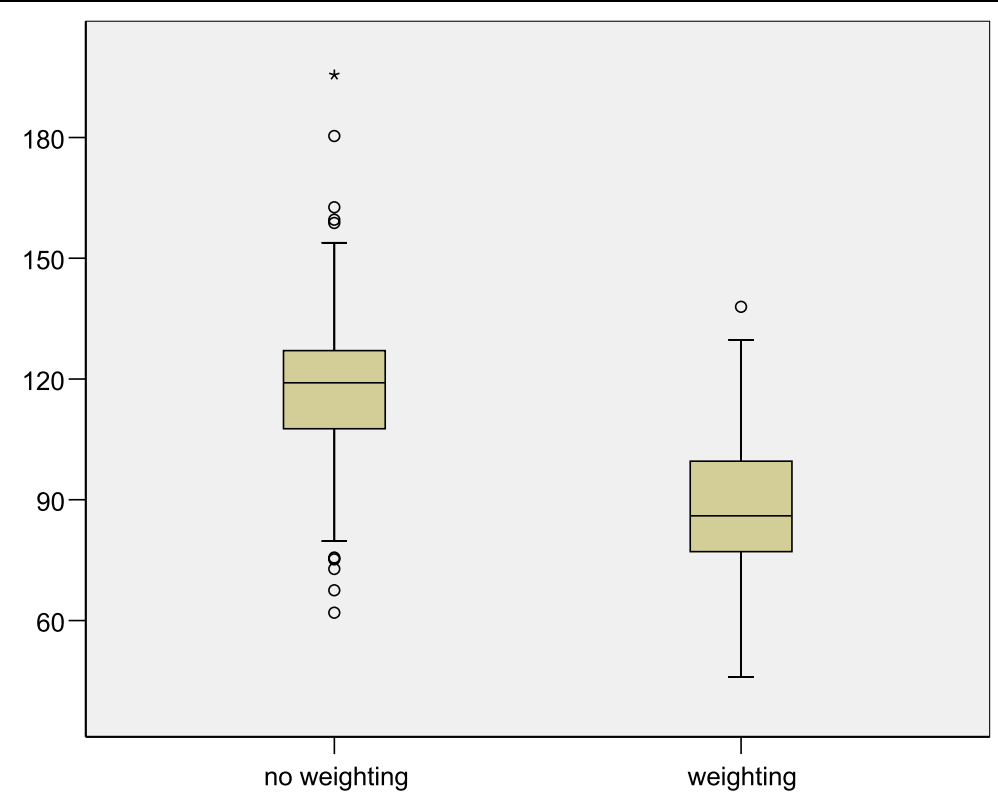

version, ${ }^{3}$ Wood's thin plate splines and the same estimator using cubic splines. Note that the modified kernel backfitting and the spline based estimators have no linear bias so that a fair comparison would have been to use the local linear smooth backfitting. However, as we have not covered the local linear version in this paper, we present a comparison based on its simple local constant version. We will see that even this simpler version still performs at least as well as its competitors.

We drew samples of size $n=500$ containing data from model (20) with $d=20, g_{j}\left(x_{j}\right)=2 \sin \left(\pi x_{j}\right)$ for all $j$, $\rho=0.1$. In the first simulation runs, the classic backfitting gave rather unsatisfactory results and even failed completely for larger $\rho$. Therefore we skipped it and only considered its modified version. Also, not surprisingly when looking at the particular curves of our simulation, cubic splines outperformed thin plate splines, and so we only present results for the cubic regression splines. For such a study it is reasonable to fix the smoothing parameters over the 1000 simulation runs. Unfortunately, it turned out that the automatic smoothing parameter selectors lead to different biases for the competitors, which is inconvenient for a direct comparison. Our cross validation criterion suggested (after 100 simulation runs) to fix $h=0.07$ for smooth backfitting. After that the other smoothing parameters were chosen accordingly to produce similar biases $(h=0.04$ for the

\footnotetext{
${ }^{3}$ Here, each function $g_{j}(x)$ is decomposed in a parametric linear part, $\beta_{j} x$, and a nonparametric component, $g_{j}^{N P}(x)$, such that $g_{j}(x)=$ $\beta_{j} x+g_{j}^{N P}(x)$. The modified backfitting then first searches for the parametric solution and only fits the remaining parts nonparametrically resulting in a strongly less volatile estimate.
}

modified classical backfitting, and $\lambda=0.2$ for the cubic splines).

As can be seen from Fig. 5, the 95\% confidence intervals (simulated from 300 repetitions) look rather similar with a slight advantage for smooth backfitting. The nearest competitor is the cubic spline based procedure. However, for the functions considered, with the range $[-1,1]$, it is clear that cubic splines have a natural advantage. It is therefore surprising (Fig. 5) how badly they perform outside the intermediate range, say outside the interval $[-0.75,0.75]$ in each dimension. We conjecture that a local linear smooth backfitting will outperform these competitors clearly but at a computational cost, compare Nielsen and Sperlich (2005) and Roca-Pardiñas and Sperlich (2008).

\subsection{Real data examples}

\subsubsection{Predicting air pollution}

The Spanish Environmental Protection Act has defined a statutory threshold, let us call it $r$, for the average emission of $\mathrm{SO}_{2}$ over two hours. This was measured every 5 minutes at ground level. The exceeding of this threshold is called a "pollution period". We attempt to estimate the probability of the threshold being exceeded within one hour. This can be used as an indicator, one hour in advance, that, in the absence of intervention, pollution is imminent. To this aim we considered a series of binary variables indicating whether the threshold has been exceeded or not, together with the lagged observations of $\mathrm{SO}_{2}$ emission taken in the surroundings of the coal/oil-fired As Pontes power station in Galicia, Spain. In other words, for $X_{t}$ denoting the value obtained by 
Fig. 5 Data generating

functions and point-wise $95 \%$ confidence bands of the estimates $\hat{g}_{j}, j=1,10,15,20$. Estimates for model (20) with $d=20, n=500, \rho=0.1$ and $g_{j}\left(x_{j}\right)=2 \sin \left(\pi x_{j}\right)$ for $j=1, \ldots, 20$. true $=$ true function, cubic $=$ cubic splines, $\mathrm{sb}=$ smooth backfitting, $\mathrm{cb}=$ modified classic backfitting

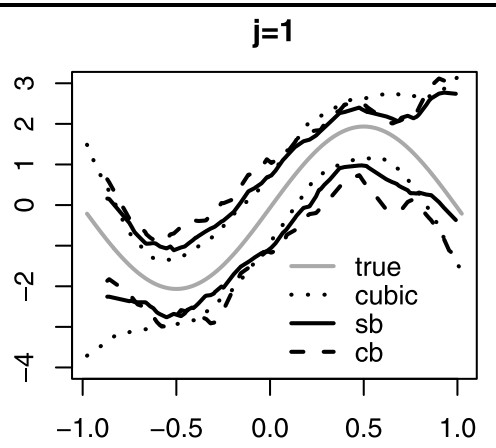

$\mathrm{j}=10$

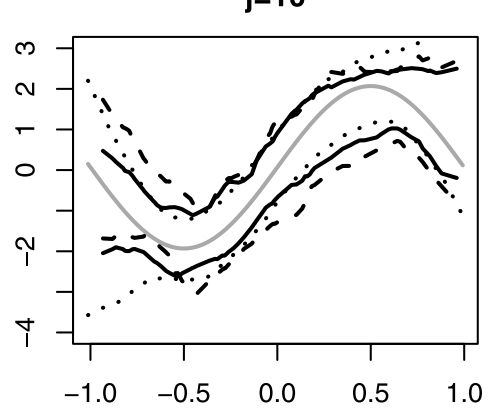

$j=15$

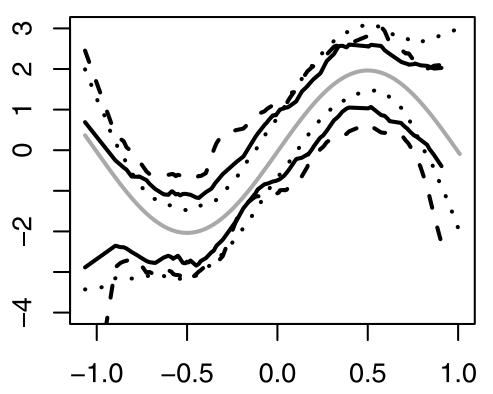

$\mathbf{j}=\mathbf{2 0}$

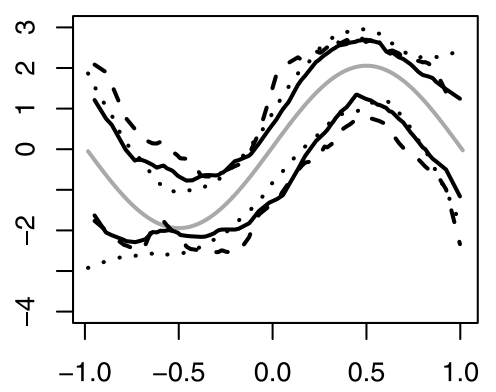

the series of bi-hourly averages for $\mathrm{SO}_{2}$ emission at time $t$, we estimated the generalized additive model

$$
\begin{aligned}
P & \left(X_{t+12}>r \mid X_{t}, \ldots, X_{t-7}\right) \\
& =\underline{G}\left\{g_{0}+g_{1}\left(X_{t}\right)+\cdots+g_{8}\left(X_{t-7}\right)\right\} .
\end{aligned}
$$

We used the data prepared by Roca-Pardiñas et al. (2004). As predictions have to be based on data containing a reasonably large number of incidents, they constructed a sample with $n=20000$ observations made before, during, and a short time after pollution periods. In the original data, most of the time, $X_{t}$ takes values close to zero, i.e. long time periods of $X_{t}=0$ are interrupted by a few hours during which values rise to high levels and then fall back to zero.

In Fig. 6 are given the estimates. For the first 6 used covariates we see a continuously increasing contamination effect, interrupted at about 250 . The larger the delay, the less slope we find, which is quite plausible. For $X_{t}$ we see further a decreasing effect at about 380 . The reason for the downslope shapes is the following: the current policy of the power station allots two interventions at different contamination levels causing two types of pollution periods (low and high), where the first intervention can be seen clearly in all $g_{j}$ whereas the high level intervention is only noticeable in $g_{1}$, owing to the immediate effectiveness of this (second) intervention. In Fig. 7 we see a typical example with both types of pollution periods during one selected day. Note that our objective here is forecasting, not modelling or fitting, and we therefore disregard further inference in this application.
However, it seems to us that an interesting alternative specification could bee an additive model with parametrically linked components, i.e. with $g_{j}=\alpha_{j} \cdot g\left(X_{t-j}\right)$ for a nonparametric $g, \alpha_{j} \in \mathbb{R}$, and $j=0, \ldots, 7$.

\subsubsection{Explaining postoperative infection}

In a prospective study conducted at the University Hospital of Santiago de Compostela, a total of $n=2318$ patients who underwent surgery at this center from January 1996 through March 1997 were characterized as follows: pre-operatively, in respect of a series of variables, including Gluc = plasma glucose concentration (in $\mathrm{mg} / \mathrm{dl}$ ) and Lymph $=$ lymphocytes (expressed as relative counts (\%) of the white blood cell count); and post-operatively, in respect of whether they suffered $(\mathrm{Poi}=1)$ or not post-operative infection $($ Poi $=0)$. The main goal of this analysis was to investigate the possible effects of Lymph and Gluc on risk of Poi adjusting for potential confounders, such as Age (in years) and Sex (coded as $1=$ male; $0=$ female). However, it is well known that Gluc affects Poi via strong interactions with all other covariates, basically causing slopechanges. Therefore we have fitted a logistic varying coefficient model

$P($ Poi $=1 \mid$ Lymph, Gluc, Age, Sex $)=\frac{\exp (m)}{1+\exp (m)}$

with $m=\alpha+\sum_{j=1}^{4} \alpha_{j}$ (Gluc)Lymph ${ }^{j-1}+\alpha_{5}$ (Gluc)Age + $\alpha_{6}$ (Gluc)Sex. Note that $\alpha_{1}$ represents the separable impact of Gluc. Since it would be interesting to know to what 
Fig. 6 Estimated $g_{j}$ for $j=1, \ldots, 8$ of prediction model (22)
Fig. 7 Example of both types of pollution periods, high (left) and low (right) during one selected day

$$
\hat{g}_{1}\left(X_{t}\right)
$$

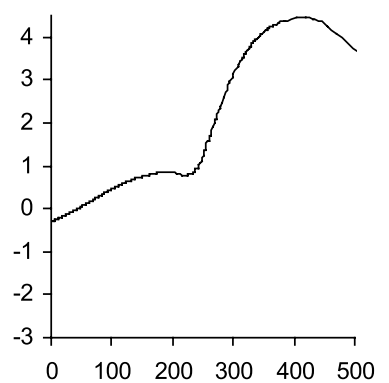

$$
\hat{g}_{4}\left(X_{t-3}\right)
$$

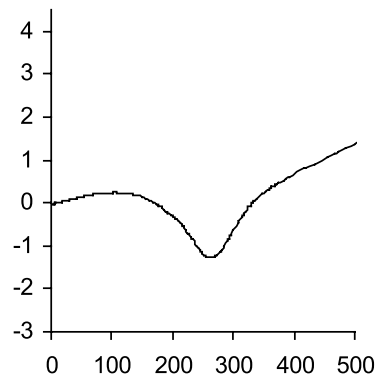

$$
\hat{g}_{7}\left(X_{t-6}\right)
$$

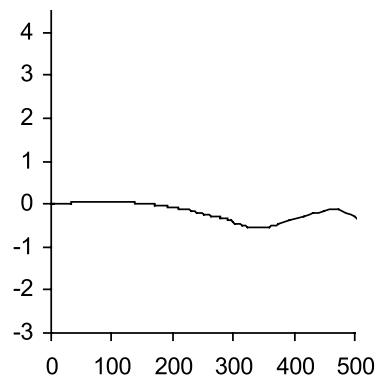

$\hat{g}_{2}\left(X_{t-1}\right)$

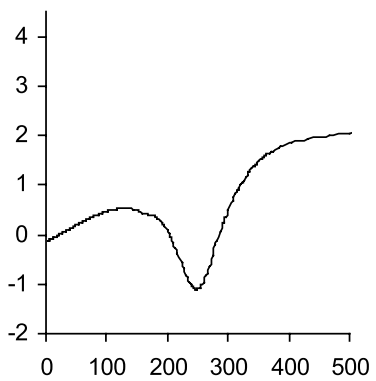

$$
\hat{g}_{5}\left(X_{t-4}\right)
$$

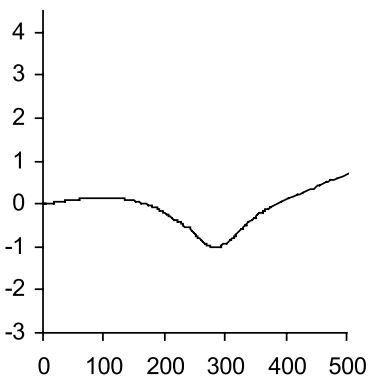

$\hat{g}_{8}\left(X_{t-7}\right)$

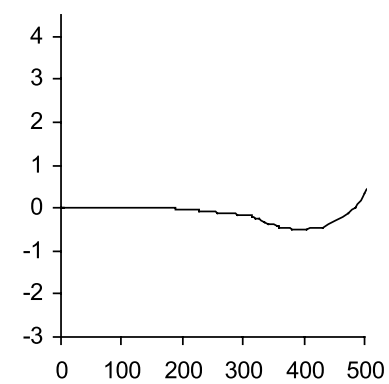

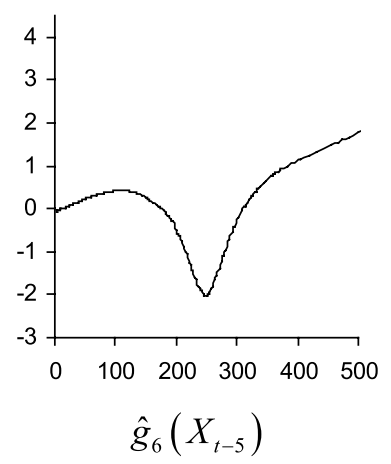

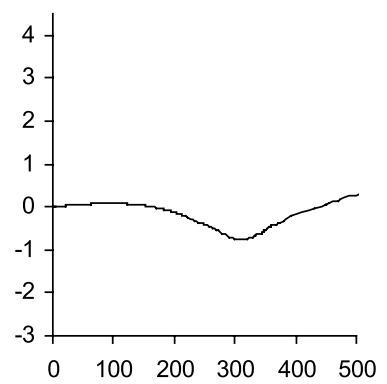

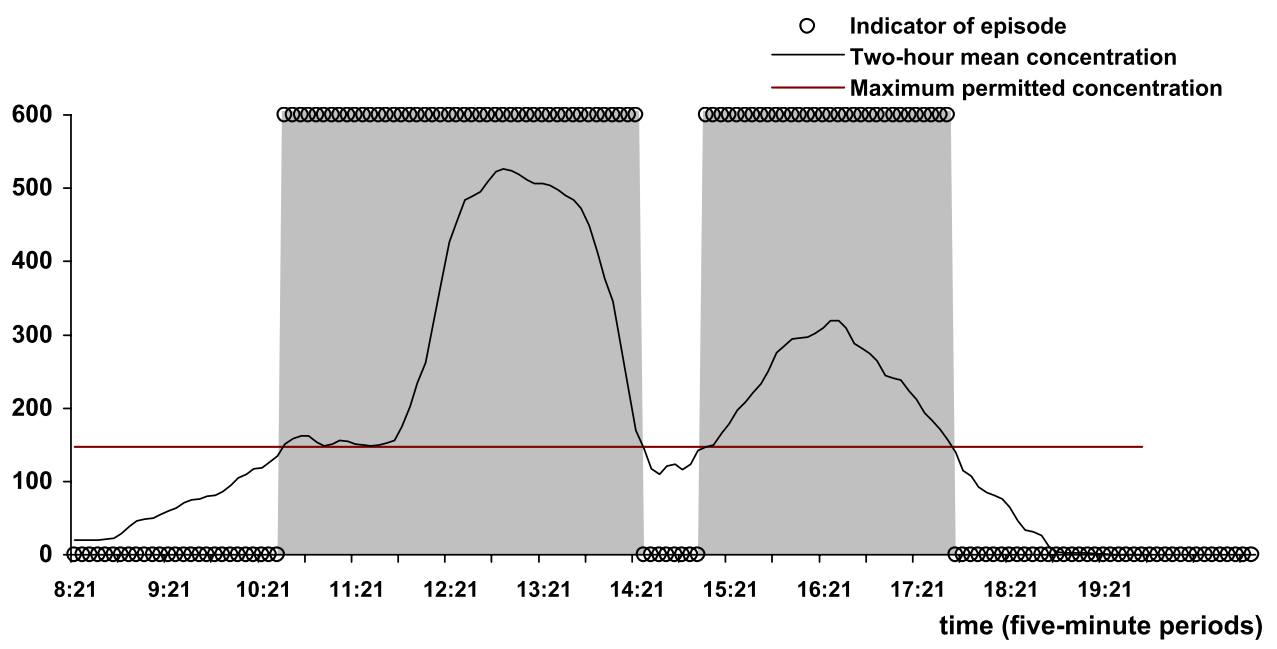

extend the parameters $\alpha_{j}, j=1, \ldots, 6$, really change with Gluc, we draw $95 \%$ point wise confidence bands. These were calculated from 300 bootstrap samples $\left\{\left(\mathrm{Poi}_{i}^{*} \mid \mathrm{Lymph}_{i}\right.\right.$,
$\left.\left.\operatorname{Gluc}_{i}, \operatorname{Age}_{i}, \operatorname{Sex}_{i}\right)\right\}_{i=1}^{n}$ with $\operatorname{Poi}_{i}^{*} \sim \operatorname{Bernoulli}\left(\hat{P}_{i}\right)$, where $\hat{P}_{i}$ denotes the estimated probability (23) given the $i$ th observation. 
$\hat{\alpha}_{1}(G L U C)$
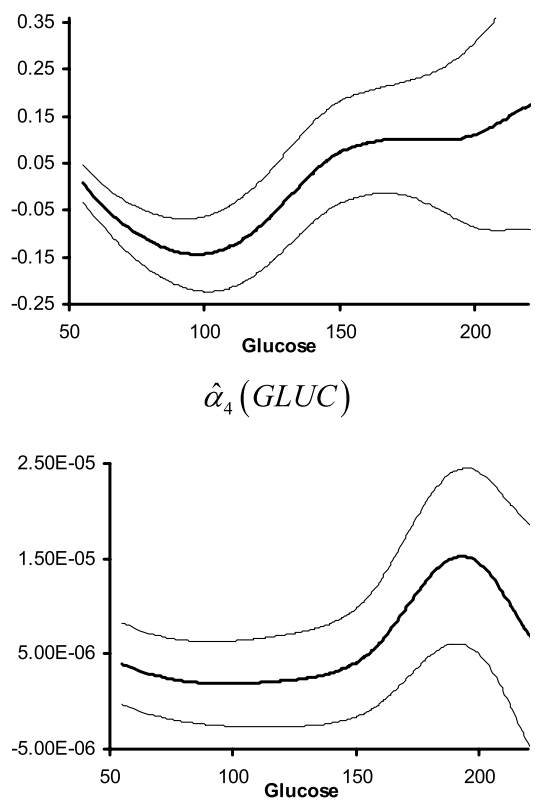

$\hat{\alpha}_{2}(G L U C)$

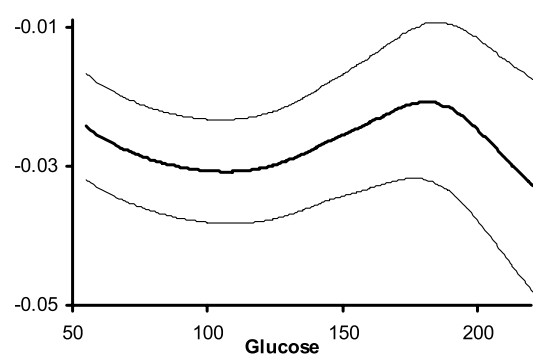

$\hat{\alpha}_{5}(G L U C)$

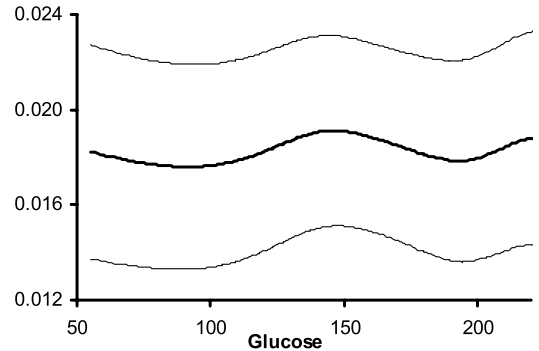

$\hat{\alpha}_{3}(G L U C)$
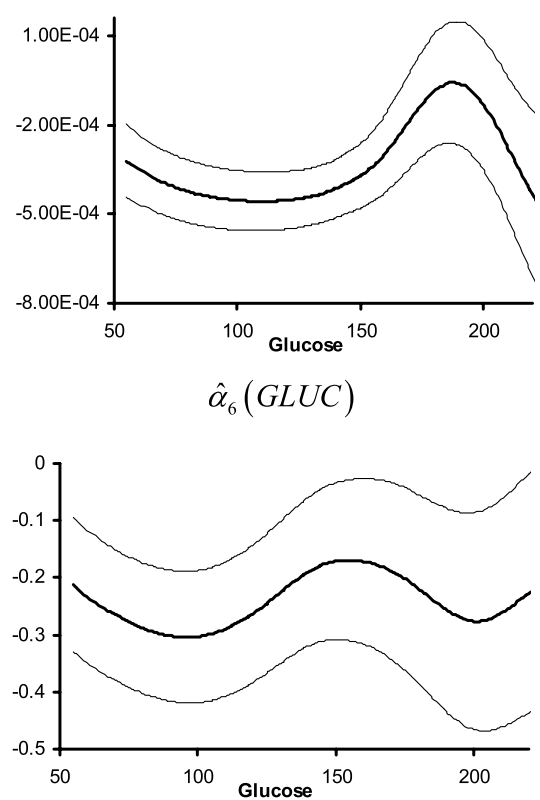

Fig. 8 Estimated $\alpha_{j}$ curves for $j=1, \ldots, 6$ obtained from model (23)

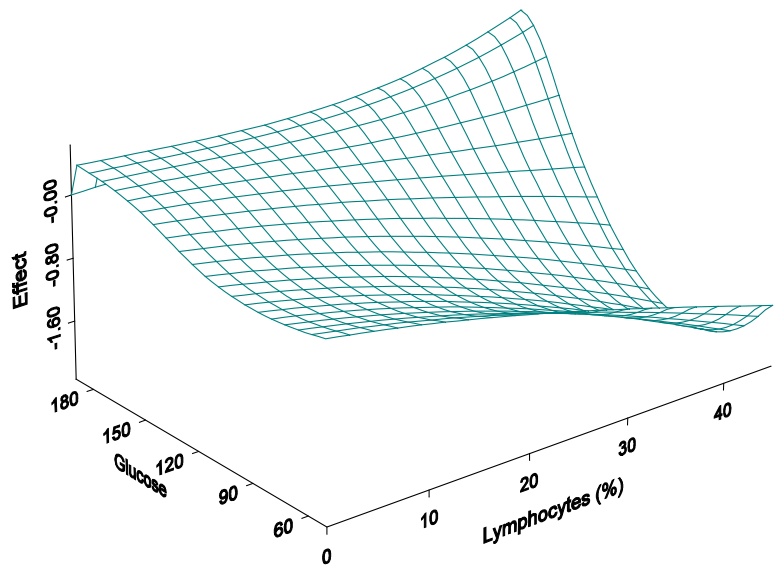

Fig. 9 Estimated Lymph-Gluc effect under model (23)

Figures 8 and 9 show that the effects of Lymph, Age and Sex depend on the level of Gluc. In particular, for low levels of Gluc, Lymph decreases the Poi risk, whereas for high Gluc-levels it is vice verse. Most coefficients $\alpha_{j}$ seem to vary over Gluc, often U-shaped, whereas $\alpha_{5}$ and $\alpha_{6}$ could be modelled as constants as well. For further discussion see Cadarso-Suárez et al. (2006), where these data were fitted with a particular generalized additive interaction model based on a kind of classical backfitting and bivariate local linear kernel smoothers. We consider our generalized varying coefficient model to be more appropriate in two ways: it has a clear interpretable and intuitive structure, and it has a lower dimensionality. Apart from that, the statistical behav-

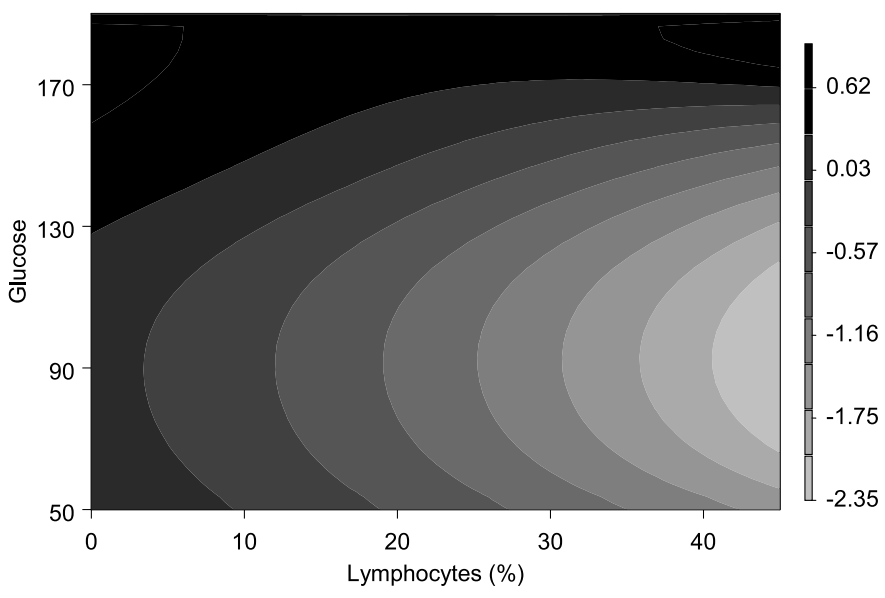

ior of their estimator is unknown but supposed to be less efficient, compare Mammen and Nielsen (2003) and Opsomer (2000).

\section{Conclusions}

We have introduced and studied a new, feasible estimation procedure for a rather general class of models which covers a good bunch of models typically used in applied empirical research. Our theoretical base is the smooth backfitting estimator of Mammen et al. (1999) and Mammen and Nielsen (2003). Our objective function (9) directly enables us to es- 
timate all models mentioned above in a feasible way. This holds for both, the mean and the variance function. Furthermore, for this estimator exists a feasible version to get more efficient estimates. Different to most of the competing estimators (if exist at all), a theoretical framework for the statistical behavior is given, indicating good performance in rate, variance and (oracle) bias. The use and excellent performance of our method are demonstrated in various simulation studies. The usefulness and need is discussed in different examples and illustrated in two real data applications from environmetrics and biometrics.

Open Access This article is distributed under the terms of the Creative Commons Attribution Noncommercial License which permits any noncommercial use, distribution, and reproduction in any medium, provided the original author(s) and source are credited.

\section{References}

Binder, H., Tutz, G.: A comparison of methods for the fitting of generalized additive models. Stat. Comput. 18, 87-99 (2008)

Cadarso-Suárez, C., Roca-Pardiñas, J., Figueiras, A.: Effect measures in nonparametric regression with interactions between continuous exposures. Stat. Med. 25, 603-621 (2006)

Fan, J., Marron, J.S.: Fast implementations of nonparametric curve estimators. J. Comput. Graph. Stat. 3, 35-56 (1994)

Friedman, J.H., Stuetzle, W.: Projection pursuit regression. J. Am. Stat. Assoc. 76, 817-823 (1981)

Härdle, W., Huet, S., Mammen, E., Sperlich, S.: Bootstrap inference in semiparametric generalized additive. Econom. Theory 20, 265300 (2004)

Hastie, T.J., Tibshirani, R.J.: Generalized Additive Models. Chapman \& Hall, London (1990)

Linton, O., Sperlich, S., Van Keilegom, I.: Estimation of a semiparametric transformation model. Ann. Stat. 36, 686-718 (2008)

Mammen, E., Nielsen, J.P.: Generalised structured models. Biometrika 90, 551-566 (2003)

Mammen, E., Linton, O., Nielsen, J.: The existence and asymptotic properties of a backfitting projection algorithm under weak conditions. Ann. Stat. 27, 1443-1490 (1999)

Mammen, E., Støve, B., Tjøstheim, D.: Nonparametric additive models for panels of time series. Presentation at the International Conference on Time Series Econometrics, Finance and Risk, Perth, June 2006

McCullagh, P., Nelder, J.A.: Generalized linear models. Chapman \& Hall, London (1989)

Nielsen, J.P., Linton, O.B.: An optimization interpretation of integration and backfitting estimators for separable nonparametric models. J. R. Stat. Soc. B 60, 217-222 (1998)
Nielsen, J.P., Sperlich, S.: Smooth backfitting in practice. J. R. Stat. Soc. B 67, 43-61 (2005)

Opsomer, J.D.: Asymptotic properties of backfitting estimators. J. Multivar. Anal. 73, 166-179 (2000)

Opsomer, J.D., Ruppert, D.: Fitting a bivariate additive model by local polynomial regression. Ann. Stat. 25, 186-211 (1997)

Roca-Pardiñas, J., Sperlich, S.: Testing the link when the index is semiparametric - a comparison study. Comput. Stat. Data Anal. 12, 6565-6581 (2007)

Roca-Pardiñas, J., Sperlich, S.: Estimating generalized structured models - a computational note. Discussion Paper, Georg August Universität Göttingen, Germany (2008)

Roca-Pardiñas, J., González-Manteiga, W., Febrero-Bande, W., PradaSánchez, J.M., Cadarso-Suárez, C.: Predicting binary time series of $\mathrm{SO}_{2}$ using generalized additive models with unknown link function. Environmetrics 15, 1-14 (2004)

Rodríguez-Poó, J.M., Sperlich, S., Vieu, P.: Semiparametric estimation of weak and strong separable models. Econom. Theory 19, 10081039 (2003)

Rodríguez-Poó, J.M., Sperlich, S., Vieu, P.: An adaptive specification test for semiparametric models. SSRN paper, available at: http://papers.ssrn.com/sol3/papers.cfm?abstract_id=1010933 (2007)

Schienle, M.: A projection approach to additive nonparametric estimation of recurrent Markov processes. Presentation at Oberwolfach Meeting, March 2007

Sperlich, S., Linton, O., Härdle, W.: Integration and backfitting methods in additive models-finite sample properties and comparison. Test 8, 419-458 (1999)

Sperlich, S., Tjøstheim, D., Yang, L.: Nonparametric estimation and testing of interaction in additive models. Econom. Theory 18, 197-251 (2002)

Stone, C.J.: Additive regression and other nonparametric models. Ann. Stat. 13, 689-705 (1985)

Stone, C.J.: The dimensionality reduction principle for generalized additive models. Ann. Stat. 14, 590-606 (1986)

Vilar-Fernández, J.M., Francisco-Fernández, M.: Local polynomial regression smoothers with AR-error structure. Test 11, 439-464 (2002)

Wahba, G., Wang, Y., Gu, C., Klein, R., Klein, B.: Smoothing spline ANOVA for exponential families, with application to the Wisconsin epidemiological study of diabetic retinopathy. Ann. Stat. 23, 1865-1895 (1995)

Wand, M.: Fast implementation of multivariate kernel estimators. J. Comput. Graph. Stat. 3, 433-445 (1994)

Wood, S.N.: Thin plate regression splines. J. R. Stat. Soc., Ser. B 65, 95-114 (2003)

Yu, K., Park, B.U., Mammen, E.: Smooth backfitting in generalized additive models. Ann. Stat. 36, 228-260 (2008)

Xiao, Z., Linton, O., Carroll, R.J., Mammen, E.: More efficient local polynomial estimation in nonparametric regression with autocorrelated errors. J. Am. Stat. Assoc. 98, 890-992 (2003) 